\title{
Magnetostatic coupling in soft/hard biphase magnetic systems based on amorphous alloys
}

\author{
J. Torrejón ${ }^{\text {a) }}$ \\ Institute of Materials Science, CSIC, 28049 Madrid, Spain \\ L. Kraus \\ Institute of Physics, ASCR, 18221 Prague, Czech Republic \\ K. R. Pirota \\ Applied Physics Department, Autonomous University of Madrid, 28049 Madrid, Spain
}

G. Badini and M. Vázquez

Institute of Materials Science, CSIC, 28049 Madrid, Spain

(Presented on 11 January 2007; received 31 October 2006; accepted 4 December 2006; published online 18 April 2007)

\begin{abstract}
We report on magnetic bias in biphase systems arising from by dipolarlike interaction. Composites consist of amorphous ribbons and wires prepared by rapid solidification techniques, surrounded by an electroplated layer. Amorphous metallic core is $\left(\mathrm{Co}_{0.94} \mathrm{Fe}_{0.06}\right)_{72.5} \mathrm{Si}_{12.5} \mathrm{~B}_{15}$ with ultrasoft magnetic behavior (coercivity of $10-10^{-1} \mathrm{~A} / \mathrm{m}$ ), and the electroplated coating is crystalline $\mathrm{Co}_{85} \mathrm{Ni}_{15}$ alloy with harder magnetic character (coercivity of $10^{4} \mathrm{~A} / \mathrm{m}$. After premagnetizing in a dc saturating field $\left(10^{6} \mathrm{~A} / \mathrm{m}\right)$, the low-field $\left( \pm 10^{3} \mathrm{~A} / \mathrm{m}\right)$ hysteresis loops are ascribed to the magnetization process of the soft core and exhibit a typical shift towards the direction of the premagnetizing field. This shift is ascribed to the magnetostatic field generated by uncompensated poles at the edges of the hard layer, which remains close to its remanence state. The influence of the geometry of both phases on magnetostatic energy term allows us to tailor the bias field and susceptibility of the core. These results open other possibilities to use these materials as sensing elements in magnetic sensor. (c) 2007 American Institute of Physics. [DOI: 10.1063/1.2710545]
\end{abstract}

Magnetic bias is a powerful tool for obtaining unique properties of multicomponent magnetic systems of technological interest in spin-valve reading heads, magnetic biosensors, random access memories, ${ }^{1}$ security tags, ${ }^{2}$ or giantmagnetoimpedance valves. ${ }^{3}$

In multilayered and nanogranular systems, three main types of magnetic interactions can be distinguished: (a) the exchange coupling between touching (ferro-/antiferro- or ferro-/ferromagnetic) phases, (b) Indirect Ruderman-KittelKasuya-Yosida (RKKY)-like coupling through an interfacial nonmagnetic metallic layer, and (c) long-range dipolarlike coupling. The main features of magnetic bias are its unidirectional anisotropy and the shift of hysteresis loop away from the zero field axis. ${ }^{4}$ The magnitude of this shift is labeled as bias field $H_{b}$, whose direction can be either parallel or antiparallel to the "frozen-in" spin direction of the pinning phase (antiferromagneticlike or hard ferromagneticlike, respectively).

In this work the magnetostatic bias phenomenon is investigated in biphase magnetic systems: bilayer ribbons and multilayer microwires. These multilayer systems are composed of an amorphous nucleus with ultrasoft magnetic character and of a crystalline harder magnetic outer shell. The amorphous nucleus is fabricated by rapid solidification techniques onto which the outer shell is electroplated. In the multilayer microwires, the two magnetic layers are separated

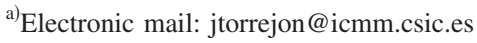

by nonmagnetic intermediate layers. In both materials, the magnetostatic coupling has been analyzed as a function of the dimensions of the two magnetic layers.

The composition of precursor polycrystalline mother alloy, $\left(\mathrm{Co}_{0.94} \mathrm{Fe}_{0.06}\right)_{72.5} \mathrm{Si}_{12.5} \mathrm{~B}_{15}$, has been selected because of its vanishing magnetostriction constant, $1 \times 10^{-7}$, which results in an ultrasoft magnetic character of the final amorphous component. Planar flow casting has been used to prepare amorphous ribbons of $0.12 \mathrm{~cm}$ wide and $20 \mu \mathrm{m}$ thick. On the other hand, quenching and drawing method has been used to fabricate glass-coated microwires, with $17.4 \mu \mathrm{m}$ diameter for the inner metallic nucleus and $12.1 \mu \mathrm{m}$ thick Pyrex glass coating. The hard magnetic outer shell, CoNi, has been electroplated directly onto the amorphous ribbons using an aqueous solution and electrolytic cell as earlier reported. ${ }^{5}$ In the case of the glass-coated microwires, a $30 \mathrm{~nm}$ thick Au nanolayer was first sputtered onto the Pyrex, which played the role of electrode/substrate for the final electroplating of the magnetically harder $\mathrm{CoNi}$ outer microlayer. The thickness and composition of the CoNi layer depend on the current density and the time of electroplating. ${ }^{6}$ A current density of $12 \mathrm{~mA} / \mathrm{cm}^{2}$ was used in the present experiments for which the composition of plated alloy was $\mathrm{Co}_{85} \mathrm{Ni}_{15}$, exhibiting hcp crystalline structure as deduced from x-ray diffraction.

Two series of hysteresis loops with the applied field parallel to the microwire/ribbon axis have been analyzed in detail: (i) high-field $( \pm 40 \mathrm{kA} / \mathrm{m}$ maximum applied field) hys- 


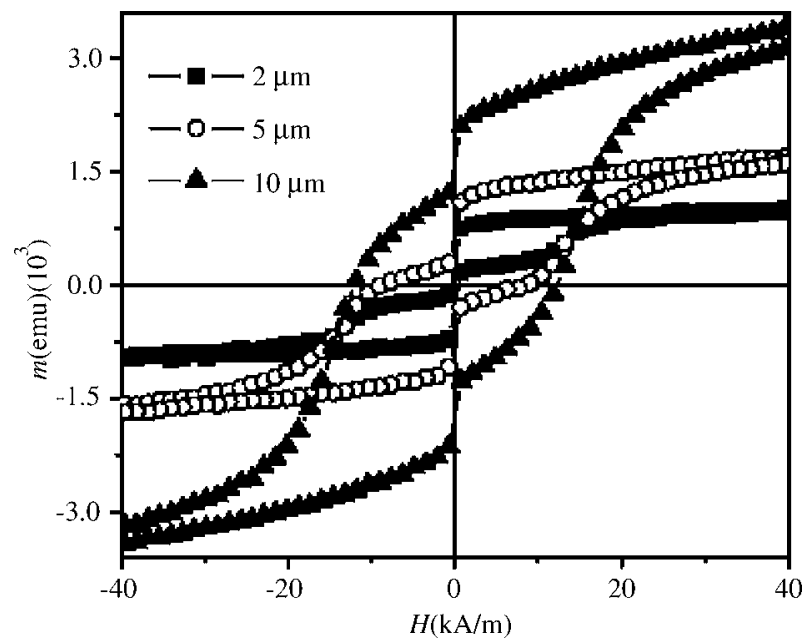

FIG. 1. High-field hysteresis loop for multilayer magnetic microwires with different CoNi thicknesses.

teresis loops using a vibrating sample magnetometer (VSM) and (ii) low-field $( \pm 500 \mathrm{~A} / \mathrm{m}$ maximum applied field) hysteresis loops measured after premagnetizing under a nearly saturating field of $\pm 40 \mathrm{kA} / \mathrm{m}$ using a high sensitivity inductive ac hysteresis loop tracer with a $0.8 \mathrm{~cm}$ long pickup coil. ${ }^{7}$

The high-field hysteresis loops of the biphase systems have been analyzed. The magnetization process takes places in two steps denoting its biphase magnetic character: (i) a giant Barkhausen jump at low very field (few $\mathrm{A} / \mathrm{m}$ ) that must be ascribed to the magnetization reversal of the amorphous nucleus and (ii) an additional jump (spread in the range between 10 and $20 \mathrm{kA} / \mathrm{m}$ ) corresponding to the harder CoNi outer shell. Figure 1 shows the high-field hysteresis loops of biphase multilayer magnetic microwires with different $\mathrm{CoNi}$ thicknesses $t_{\mathrm{CoNi}}$. The lengths of both phases, the soft nucleus, $L_{s}$, and the CoNi outer shell, $l_{\mathrm{CoNi}}$, were $5 \mathrm{~mm}$. As observed, coercivity, remanence, and saturation magnetization of the biphase magnetic system increase with the CoNi thickness.

Figure 1 does not offer information about the possible magnetic interaction between the two magnetic phases. To investigate such interaction, the following two-step process has been considered: (a) The biphase samples are premagnetized under a nearly saturating dc magnetic field $( \pm 40 \mathrm{kA} / \mathrm{m})$ and (b) the hysteresis loop is then analyzed at low field under a maximum applied field large enough to reverse the magnetization of the soft phase $( \pm 500 \mathrm{~A} / \mathrm{m})$ but sufficiently small to keep the hard phase close to its remanence state far from its irreversible magnetization processes. In this case, only the internal nucleus contributes to the low-field hysteresis loops.

Figure 2 shows the low-field hysteresis loop of a multilayer microwire magnetic microwire with a CoNi thickness $t_{\mathrm{CoNi}}$ of $5 \mu \mathrm{m}$ (its high-field loop is given in Fig. 1), measured after premagnetizing at $\pm 40 \mathrm{kA} / \mathrm{m}$. One can observe that (a) the loops are shifted towards the orientation of the premagnetizing field by a bias field of $\pm 55 \mathrm{~A} / \mathrm{m}$ and (b) both loops exhibit very modest hysteresis.

A similar effect is observed for bilayer ribbons. Figure 3 shows the low-field hysteresis loops of bilayer ribbons with different CoNi thicknesses, after premagnetizing at

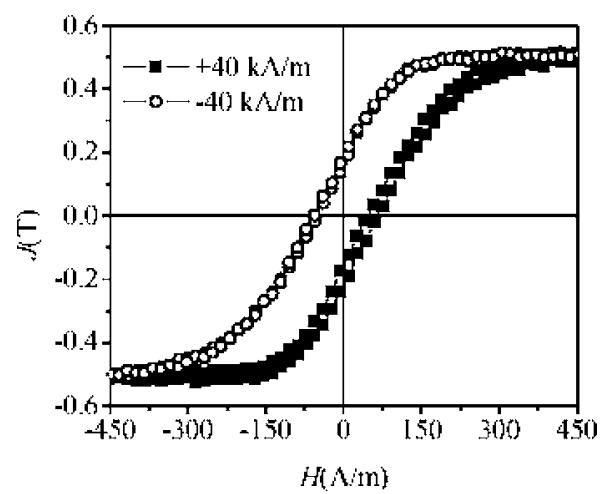

FIG. 2. Low-field hysteresis loops for a multilayer magnetic microwire with CoNi thickness of $5 \mu \mathrm{m}$, after premagnetizing at $\pm 40 \mathrm{kA} / \mathrm{m}$.

$+40 \mathrm{kA} / \mathrm{m}$. Now, the lengths of the nucleus, $L_{s}$, and the CoNi outer shell, $l_{\mathrm{CoNi}}$, are 4 and $2 \mathrm{~cm}$, respectively. As observed, the bias field increases with CoNi thickness, and for the larger values two slopes in the magnetization process of the nucleus can be identified. This indicates the presence of two regions within the soft magnetic phase with different susceptibility: (a) region 1 with higher susceptibility $\chi_{1}$ and given fractional volume $V_{1}$, which would give rise to a bias field $H_{b 1}$, and (b) region 2 with smaller susceptibility $\chi_{2}$, bias field $H_{b 2}$, and fractional volume $V_{2}$. The evolution of susceptibilities and fractional volumes can be followed in Fig. 3: a monotonic decrease of $V_{1}$ and parallel increase of $V_{2}$ with the CoNi thickness are observed. Consequently, the thickness of the outer shell actually determines $V_{1}$ and $V_{2}$. The inset in Fig. 3 shows the dependence of the bias field on the thickness of the hard layer, $t_{\mathrm{CoNi}}$. As observed, the bias field increases nearly linearly until the thickness reaches a value of $18 \mu \mathrm{m}$. Along this linear region, $V_{1}>V_{2}$, and $H_{b}$ is mainly determined by region 1 with higher susceptibility. For larger thickness, the linear behavior is broken due to $V_{1}<V_{2}$ and $H_{b}$ is mainly controlled by the region 2 .

The shift of the low-field hysteresis loops for biphase ribbons and wires is in the same direction as that of the previously applied saturating field. It shows that $H_{b}$ is opposite to the remanence of $\mathrm{CoNi}$, which denotes the antiferromagneticlike character of the bias coupling. This kind of interaction can be expected for a short-range antiferromagnetic contact exchange coupling at the hard/soft magnetic

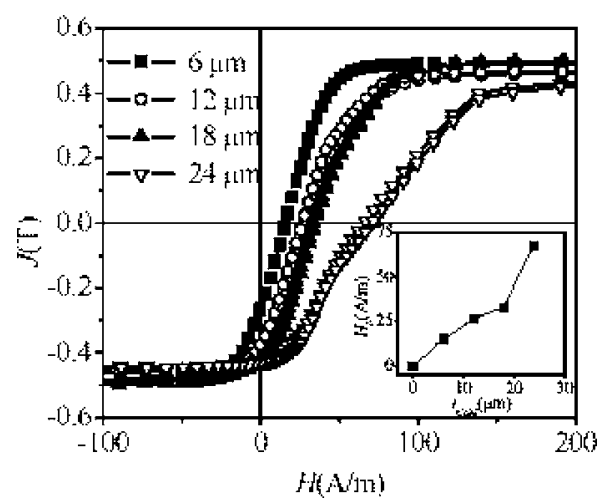

FIG. 3. Low-field hysteresis loops of bilayer ribbons with different $t_{\mathrm{CoNi}}$, after premagnetizing at $+40 \mathrm{kA} / \mathrm{m}$. Inset: the bias field dependence on the CoNi thickness. 


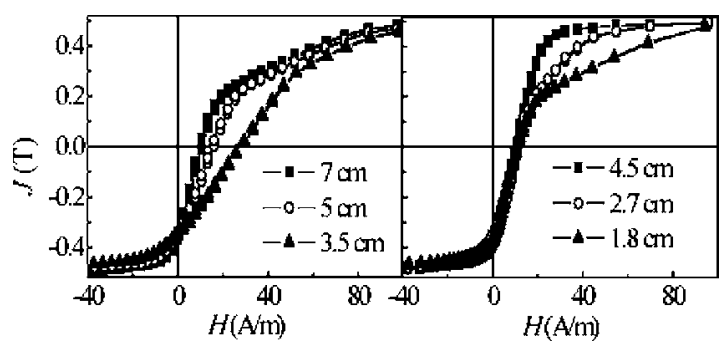

FIG. 4. Low-field loops of bilayers ribbons, measured after premagnetizing under $+40 \mathrm{kA} / \mathrm{m}$ for a range of different lengths of the (a) soft layer ( $\left.l_{\mathrm{CoNi}}=1.8 \mathrm{~cm}, t_{\mathrm{CoNi}}=12 \mu \mathrm{m}\right)$ and of $(\mathrm{b})$ the hard layer $\left(L_{s}=7 \mathrm{~cm}, t_{\mathrm{CoNi}}\right.$ $=12 \mu \mathrm{m})$.

interface or for a long-range magnetostatic coupling of the soft magnetic phase with the stray fields of magnetic charges on the hard magnetic layers. The real origin can be determined from an analysis of the dependence of the bias field on the CoNi thickness. It is known that the exchange bias in FM/AFM bilayers does not depend on the AFM layer thickness for thickness above a few nanometers. ${ }^{8}$ Figure 3 shows, however, that the bias field is proportional to the micrometric thickness of the hard magnetic layer, which indicates that magnetostatic coupling is responsible for the magnetic bias. Moreover, in the case of the multilayer magnetic microwires, exchange bias coupling must be straightforwardly discarded because of the presence of nonmagnetic intermediate microlayers.

The origin of the magnetostatic coupling can be understood as follows: the nearly saturating premagnetizing field creates net magnetic charges at the ends of the external $\mathrm{CoNi}$ layer, which remain unbalanced and create an antiparallel magnetostatic field $H_{b}$ in the soft amorphous layer. When the CoNi thickness of the hard phase is large enough, the magnetization reversal of the soft nucleus is characterized by two regions with different susceptibilities and bias fields, due to the fact that internal magnetic field generated by the hard phase is not homogeneous.

On the other hand, as a consequence of the magnetostatic character of the coupling, the length of the different magnetic layers should play an important role. So, further experiments with different lengths of the layers (with constant CoNi thickness) have been performed to obtain detailed information on the magnetic interaction between soft and hard layers. Figure 4(a) shows the biased low-field loops of the soft layer ribbons with length $L_{s}$ ranging between 7 and $3.5 \mathrm{~cm}$, after premagnetizing at $+40 \mathrm{kA} / \mathrm{m}$. The length $l_{\mathrm{CoNi}}$, and thickness $t_{\mathrm{CoNi}}$, of hard layers remain constant at $1.8 \mathrm{~cm}$ and $12 \mu \mathrm{m}$, respectively. Again, the loops for all the samples are characterized by two regions with different susceptibilities. Note that the susceptibility in the lower-field region $\chi_{1}$ decreases when the length of the soft layer decreases, which seemingly derives from the increase of the demagnetizing field of the nucleus that also gives rise to an effective increase of the bias field. The susceptibility of the higher-field region $\chi_{2}$ remains practically unchanged with the modification of $L_{s}$.

In turn, Fig. 4(b) shows the low-field loops as a function of the length $l_{\mathrm{CoNi}}$, for constant values $L_{s}=7 \mathrm{~cm}$ and $t_{\mathrm{CoNi}}$
TABLE I. Values of $V_{1}(\%), V_{2}(\%), H_{b 1}(\mathrm{~A} / \mathrm{m}), H_{b 2}(\mathrm{~A} / \mathrm{m}), \chi_{1}$, and $\chi_{2}$ for bilayer ribbons with different dimensions: $t_{\mathrm{CoNi}}(\mu \mathrm{m}), L_{s}(\mathrm{~cm})$, and $l_{\mathrm{CoNi}}$ (cm).

\begin{tabular}{cllrrrrrr}
\hline \hline$t_{\mathrm{CoNi}}$ & $L_{s}$ & $l_{\mathrm{CoNi}}$ & $V_{1}$ & $V_{2}$ & $H_{b 1}$ & $H_{b 2}$ & $\chi_{1}$ & $\chi_{2}$ \\
\hline 6 & 4 & 2 & 100 & 0 & 15 & 0 & 9680 & 0 \\
12 & 4 & 2 & 71 & 29 & 18 & 63 & 9680 & 4120 \\
18 & 4 & 2 & 64 & 36 & 18.5 & 68 & 9680 & 5630 \\
24 & 4 & 2 & 35 & 65 & 23 & 89 & 7930 & 4440 \\
12 & 3.5 & 1.8 & 80 & 20 & 22.5 & 70 & 8570 & 3330 \\
12 & 5 & 1.8 & 73 & 27 & 13.5 & 55 & 17460 & 3170 \\
12 & 7 & 1.8 & 70 & 30 & 7.5 & 45 & 22.000 & 3250 \\
12 & 7 & 2.7 & 72 & 28 & 8.4 & 38 & 22.000 & 9840 \\
12 & 7 & 4.5 & 100 & 0 & 10.7 & 0 & 22.000 & 0 \\
\hline \hline
\end{tabular}

$=12 \mu \mathrm{m}$. It can be observed that the two-step behavior appears only for the shortest hard layers. Now, the susceptibility of the higher-field region $\chi_{2}$, decreases when the length of hard phase is reduced, as a consequence of the increase of demagnetizing field of the hard magnetic outer shell, while $\chi_{1}$ remains constant. Thus, it can be concluded that $L_{s}$ determines the susceptibility of region 1 , while $l_{\mathrm{CoNi}}$ does so with the susceptibility of region 2 . The reason why $H_{b}$ only changes with $L_{s}$ is ascribed to the fact that all samples in Figs. 4(a) and 4(b) exhibit $V_{1}>V_{2}$, and $H_{b}$ is mainly determined by region 1 . Finally, the values of fractional volume, bias field, and susceptibility of each region $\left(V_{1}, V_{2}, H_{b 1}, H_{b 2}\right.$, $\chi_{1}$, and $\chi_{2}$ ) for bilayer ribbons of Figs. 3 and 4 are collected in Table I.

The magnetostatic coupling has been analyzed for biphase systems (ribbons and microwires) with soft/hard magnetic behavior: a soft nucleus and a hard outer shell. The magnetostatic interaction of antiferromagneticlike character is ascribed to uncompensated poles at the ends of the hard layer. Moreover, two magnetic regions with different susceptibilities and fractional volumes have been identified within the soft nucleus. Finally, the relative geometry dimensions of the layers determine the described characteristics of the loops, which offer an additional interest of these materials to be used as sensing elements in sensor field devices.

The work has been supported by the Czech research Project No. AVOZ10100520 [for one of the authors (L.K.)] and the Spanish Research Project No. MAT2004-00150. Another authors (J. T.) wishes to thank FPU program fellowship from MEC.

${ }^{1}$ J. Nogués, J. Sort, V. Langlais, V. Skumryev, S. Suriñach, J. S. Muñoz, and M. D. Baró, Phys. Rep. 422, 65 (2005).

${ }^{2}$ K. H. Shin, C. D. GrahamJr., and P. Y. Zhou, J. Magn. Magn. Mater. 177-181, 225 (1998).

${ }^{3}$ C. G. Kim, K. J. Jang, H. C. Kim, and S. S. Yoon, J. Appl. Phys. 85, 5447 (1999).

${ }^{4}$ W. H. Meiklejohn and C. P. Bean, Phys. Rev. 105, 904-913 (1957).

${ }^{5}$ K. Pirota, M. Hernández-Vélez, D. Navas, A. Zhukov, and M. Vázquez, Adv. Funct. Mater. 14, 266 (2004).

${ }^{6}$ K. Pirota, M. Provencio, K. García, P. Mendoza, M. Hernandez-Velez, and M. Vazquez, J. Magn. Magn. Mater. 290, 68 (2005).

${ }^{7}$ L. Kraus and O. Chayka, J. Electr. Eng. (to be published).

${ }^{8}$ J. Nogués and I. K. Schuller, J. Magn. Magn. Mater. 192, 203 (1999). 